\title{
Incorporação das tecnologias de informação e comunicação na integração ensino-serviço dos cursos de saúde de uma universidade pública
}

\author{
Emille Raíza Luna Gomes Ramos*; Fábio Barbosa de Souza**; Márcia Maria Dantas Cabral \\ de Melo*** \\ * Cirurgiã-dentista graduada pela Universidade Federal de \\ Pernambuco \\ ** Doutor em Dentística, Professor Adjunto, Departamento de Prótese \\ e Cirurgia Bucofacial, Universidade Federal de Pernambuco \\ *** Doutora em Saúde Coletiva, Professora Adjunta Departamento de \\ Clínica e Odontologia Preventiva, Universidade Federal de \\ Pernambuco
}

Recebido em 14/12/2017. Aprovado em 29/07/2018.

\begin{abstract}
RESUMO
Com o objetivo de analisar a incorporação de Tecnologias de Informação e Comunicação (TICs) pelos docentes dos nove cursos de saúde da Universidade Federal de Pernambuco (UFPE) nas atividades de integração ensino-serviço nos Estágios Curriculares Supervisionados (ECS), realizouse um estudo observacional e descritivo com abordagem quantitativa. A amostra foi constituída pelos coordenadores de curso e de estágios, assim como por professores supervisores dos ECS dos referidos cursos (27). Para a coleta dos dados, utilizou-se formulário de entrevista submetido a validação de face e aplicado face a face. Todos os 27 docentes selecionados foram entrevistados, sendo 92,6\% mulheres e $85,2 \%$ com doutorado. A maioria utilizava TICs $(77,8 \%)$ nos ECS, mas observou-se diferenças quanto à mídia escolhida $(\mathrm{p}=0,032)$. O Facebook $(63,0 \%)$ foi a TIC com maior preferência e mais utilizada pelos professores $(88,9 \%)$; seguido por e-mail $(59,3 \%)$ e WhatsApp $(37,0 \%)$. Sobre as finalidades de emprego das TICs nos ECS, predominou a comunicação com alunos e preceptores, que também foi mais realizada pelos coordenadores de estágios e professores do que pelos coordenadores dos cursos $(\mathrm{p}=0,024)$. A maioria relatou possuir domínio mediano nas TICs e apenas $18,5 \%$ obteve formação para seu uso. A maioria $(77,8 \%)$ empregava métodos de educação a distância (EaD) nos ECS, apesar da UFPE, disponibilizar plataformas institucionais. As TICs mostraram-se incorporadas com nuances variadas na integração ensino-serviço, com diferenças de aplicabilidade de acordo com a função exercida pelo docente nos estágios.

Descritores: Educação Superior. Mídias sociais. Tecnologia da Informação. Estágios. Sistema Único de Saúde.
\end{abstract}

\section{INTRODUÇÃO}

A passagem do modelo de desenvolvimento industrial para o modelo de desenvolvimento informacional provocou um intenso movimento de transformação em todas as esferas da estrutura social, política, econômica, jurídica e do trabalho ${ }^{1}$. Consequentemente, a evolução das Tecnologias da Informação e Comunicação (TICs) permitiu que a 
maioria da população tivesse acesso à informação, o que trouxe mudanças profundas, especialmente no campo acadêmico, onde são discutidos e construídos conhecimentos $^{2}$.

Mais recentemente, no âmbito das políticas indutoras para fortalecer a integração ensino-serviço e comunidade para avançar na consolidação do modelo de atenção à saúde preconizado pelo SUS, destaca-se a implantação das Diretrizes Curriculares Nacionais (DCN) para os cursos da Saúde ${ }^{3-6}$ que visam substituir o modelo de ensino conservador e instaurar um processo formativo capaz de enfrentar o perfil socioepidemiológico prevalente ${ }^{7}$. Tais mudanças exigem a incorporação de novas estratégias de ensino-aprendizagem e das TICs no seu processo de trabalho ${ }^{1,8-10}$.

Nesse contexto, os Estágios Curriculares Supervisionados (ECS) desempenham o papel de inserir o aluno no trabalho do SUS ${ }^{11}$, para desenvolver, em diversos cenários de práticas em saúde, competências para enfrentar o mundo futuro do trabalho em saúde ${ }^{12}$. Nesse novo cenário emergem as TICs, como uma potente ferramenta de integração entre os atores do serviço e do ensino ${ }^{1}$. Mendes (2008) ${ }^{13}$ define TICs como um conjunto de recursos tecnológicos que, quando integrados entre si, proporcionam a automação e/ou a comunicação nos processos existentes nos negócios, no ensino e na pesquisa científica. Discute-se que as TICs têm feito cada vez mais parte das atividades cotidianas da sociedade atual, provocando mudanças na forma de acesso à informação e no modo de estudar e aprender.

Considera-se que as TICs possuem um potencial formativo que pode contribuir para ampliação dos espaços e dos tempos pedagógicos, para a flexibilização do currículo e para o aumento da interação entre os sujeitos, tanto na educação presencial quanto na educação a distância $(\mathrm{EaD})^{14}$. Contudo, a incorporação do seu uso como ferramenta pedagógica em cursos de graduação parece ser heterogênea. No ensino de saúde, o uso das TICs vem sendo consolidado e pesquisas demonstram que os estudantes estão apresentando um desempenho acadêmico melhor quando possuem acesso ao material complementar online associado às aulas tradicionais ${ }^{15}$. Se espera que as tecnologias emergentes façam com que o ensino evolua do método tradicional centrado nas aulas do professor para um modelo novo centrado num ambiente de ensino interativo, focado agora no aluno ${ }^{16}$.

No sentido de se adequar às orientações nacionais para o ensino universitário e da Área da Saúde, a UFPE recentemente estimulou os cursos de graduação a promover reformulação dos seus currículos e implantar um novo Projeto Pedagógico, considerando o entendimento mais amplo das DCN, valorizando a formação contextualizada por meio de metodologias ativas e favorecendo a inserção precoce do aluno nos campos de práticas, como por exemplo nos ECS na rede de serviços do SUS ${ }^{5,7}$.

Nesse contexto, o presente estudo apresenta uma análise do estágio de incorporação das TICs em atividades de ECS realizadas pelos cursos de saúde da UFPE e seu uso pelos docentes.

\section{METODOLOGIA}

Este estudo define-se como um observacional e descritivo com abordagem quantitativa, direcionado aos nove cursos de graduação vinculados ao Centro de Ciências da Saúde (CCS) da UFPE (Educação Física, Enfermagem, Farmácia, Fisioterapia, Fonoaudiologia, Medicina, Nutrição, Odontologia e Terapia Ocupacional). O CCS está situado no campus universitário da UFPE, localizado na zona oeste da cidade do Recife. A população de estudo foi constituída por docentes dos referidos cursos envolvidos em ações de integração ensino-serviço nos ECS realizados na rede de atenção à saúde pública da região metropolitana do Recife. Adotou-se como critério de inclusão estar, no período da coleta de dados, no exercício da função de coordenador de curso, de coordenação de 
estágio e/ou de docente supervisor de ECS. A amostra foi constituída pela totalidade de coordenadores dos cursos (9), dos ECS (9) e por um docente envolvido em atividades de supervisão de estágio em cada curso (9), totalizando 27 participantes.

A coleta dos dados foi realizada no primeiro trimestre de 2017, por meio da aplicação de um formulário de entrevista semiestruturado e organizado em dois blocos. O primeiro, para a caracterização da amostra, incluiu variáveis sociodemográficas, de formação profissional e sobre as atividades de integração ensino-serviço existentes em cada curso. O segundo bloco reuniu variáveis para verificar o uso das TICs pelos docentes em atividades relacionadas aos ECS. O mesmo foi aplicado individualmente (face a face) aos sujeitos que aceitaram participar da pesquisa $\mathrm{e}$ assinaram o Termo de Consentimento Livre e Esclarecido. A elaboração do questionário foi precedida por revisão bibliográfica e pela análise dos Projeto Pedagógico dos cursos. Para garantir o controle de qualidade dos dados foram selecionados dois especialistas com experiência relacionada à problemática do estudo, para avaliarem o questionário com a finalidade da obtenção da validação de face do instrumento. A pesquisadora foi treinada para conduzir a coleta dos dados junto aos entrevistados ${ }^{17}$.

Os dados foram analisados descritivamente por meio da obtenção de frequências absolutas e percentuais. Foram realizadas análises exploratórias usando o teste Qui-quadrado de Pearson com correção do teste Exato de Fisher. Para essas análises foi adotado o nível de significância de 5\%. O programa estatístico utilizado foi o Statistical Package for the Social Sciences (SPSS Statistics, versão. 21.0; IBM Brasil, São Paulo, SP, Brasil).

$\mathrm{O}$ projeto de pesquisa foi aprovado pelo Comitê de Ética em Pesquisas do Centro de Ciências da Saúde da UFPE, CAAE:
63632816.3.0000.5208, Parecer 1.956.011.10.

\section{RESULTADOS}

Todos os sujeitos selecionados responderam à entrevista e tiveram seus formulários analisados. A caracterização sociodemográfica da amostra total indicou que a maioria era do sexo feminino. Apenas 2 docentes, coordenadores de curso, eram do sexo masculino. Em relação à idade, a média observada de todos os participantes foi de aproximadamente 49 anos $(48,85 \pm 10,14)$. Sobre a titulação, a maioria é graduada na área do curso no qual trabalha, com exceção de um entrevistado, coordenador dos ECS do curso de Medicina que possui graduação em Serviço Social e doutorado em Saúde Coletiva. A titulação ao nível de doutorado predominou, sendo que os professores supervisores em sua totalidade detêm este grau de formação. Sobre as atividades de integração ensino-serviço, todos os 9 cursos desenvolvem ECS na rede pública de atenção à saúde, e 5 deles também desenvolvem estágios não obrigatórios, além de outras atividades na rede de atenção (projetos Pró-PET-Saúde) e 7 cursos informaram participar de programas de Residência.

Na tabela 1 estão apresentados os resultados sobre o uso das TICs nos ECS dos cursos de saúde da UFPE, segundo o tipo de participante do estudo. Verificou-se que a maioria dos entrevistados faz uso das TICs. Porém, observou-se diferença estatisticamente significante $(\mathrm{p}=0,005)$ de acordo com a função dos participantes, uma vez que menos da metade dos coordenadores de curso informaram usar TICs. Sobre os tipos de TICs mais utilizadas nos ECS, foi verificado na amostra total a preferência pelo Facebook. Verificou-se diferença estatisticamente significante ao nível de $\mathrm{p}=0,032$ para o uso do correio eletrônico, com a segunda maior preferência de uso. $\mathrm{O}$ aplicativo WhatsApp foi o terceiro meio de comunicação mais frequentemente utilizado nas atividades de ECS da amostra total. Outros aplicativos como Youtube, Instagram, e Dropbox foram pouco mencionados. $\mathrm{O}$ 
uso do Ambiente Virtual de Aprendizagem de Medicina (AVAMED) da UFPE em parceria com o Núcleo de Telessaúde (NUTES), foi informado por apenas um entrevistado, que exerce a função de professor supervisor no ECS do curso de graduação em Medicina.

Tabela 1. Caracterização do uso de TICs nos Estágios curriculares dos cursos de saúde da UFPE, segundo a função exercida

\begin{tabular}{|c|c|c|c|c|c|c|c|c|c|}
\hline \multirow[t]{3}{*}{ Variável } & \multicolumn{8}{|c|}{ Função exercida } & \multirow{3}{*}{$\mathbf{p}$} \\
\hline & \multicolumn{2}{|c|}{$\begin{array}{l}\text { Coordenador } \\
\text { de curso }\end{array}$} & \multicolumn{2}{|c|}{$\begin{array}{l}\text { Professor } \\
\text { supervisor }\end{array}$} & \multicolumn{2}{|c|}{$\begin{array}{l}\text { Coordenador } \\
\text { de estágio }\end{array}$} & \multicolumn{2}{|c|}{ Total } & \\
\hline & n & $\%$ & n & $\%$ & n & $\%$ & $\mathbf{n}$ & $\%$ & \\
\hline Uso de TICs & & & & & & & & & $0,005^{*}$ \\
\hline Sim & 4 & 44,4 & 9 & 100 & 9 & 100 & 22 & 77,8 & \\
\hline Não & 5 & 55,6 & - & - & - & - & 8 & 22,2 & \\
\hline \multicolumn{10}{|l|}{ Tipo de TIC } \\
\hline Facebook & 3 & 33,3 & 8 & 88,9 & 6 & 66,7 & 17 & 63,0 & 0,069 \\
\hline WhatsApp & 2 & 22,2 & 4 & 44,4 & 4 & 44,4 & 10 & 37,0 & 0,684 \\
\hline E-mail & 2 & 22,2 & 7 & 77,8 & 7 & 77,8 & 16 & 59,3 & $0,032 *$ \\
\hline Instagram & 1 & 11,1 & 1 & 11,1 & - & - & 2 & 7,4 & 1,000 \\
\hline Twitter & - & & - & & - & & & & 1,000 \\
\hline Youtube & 1 & 11,1 & 1 & 11,1 & 1 & 11,1 & 3 & 11,1 & 1,000 \\
\hline DropBox & - & - & - & - & 1 & 11,1 & 1 & 3,7 & 1,000 \\
\hline Avamed** & - & - & 1 & 11,1 & - & - & 1 & 3,7 & 1,000 \\
\hline Total & 9 & 100 & 9 & 100 & 9 & 100 & 27 & 100 & \\
\hline
\end{tabular}

* Diferença significativa (5\%), teste Exato de Fisher

** Ambiente Virtual de Aprendizagem de Medicina da UFPE

A tabela 2 apresenta os resultados sobre o emprego das TICs pelos entrevistados nas suas atividades junto aos ECS. Observou-se que o uso de TICs com a finalidade de comunicação seja com os alunos e/ou preceptores dos ECS foi a predominante entre os professores e coordenadores de estágios, tendo sido verificada diferença significativa entre os tipos de entrevistados, apenas para a comunicação com os alunos $(\mathrm{p}=0,005)$. Todos professores e coordenadores de estágio se comunicavam com os alunos, enquanto apenas 4 coordenadores de curso afirmaram realizar tal comunicação. Já com os preceptores essa comunicação foi feita por $2 / 3$ dos professores e coordenadores de estágio e por $1 / 3$ dos coordenadores de curso.

A comunicação por meio de TICs, realizada entre os 3 tipos docentes durante as atividades de
ECS apresentou diferença significante $(p=0,024)$. Foi relatado pelo total da amostra dos professores supervisores haver comunicação entre eles por meio de TICs, sendo esse tipo de uso menos relatado pelos coordenadores dos cursos. A maioria dos coordenadores dos ECS afirmaram utilizar para esse fim. A comunicação realizada para resolver questões dos ECS com a Pró-Reitoria Acadêmica (PROACAD) da UFPE foi observada com maior frequência entre os coordenadores de ECS e a variação nesta frequência nas outras duas categorias não foi significativa. Contudo, observou-se diferença estatisticamente significante $(\mathrm{p}=0,024)$, entre os três tipos de docentes, quanto à divulgação pública de informações sobre os ECS. Já o uso da comunicação com TICs para o desenvolvimento de estratégias pedagógicas e em pesquisas para os ECS foi mais relatado pelos professores supervisores. 
Tabela 2. Uso de TICs nos estágios curriculares dos cursos de saúde da UFPE, segundo a função exercida

\begin{tabular}{|c|c|c|c|c|c|c|c|c|c|}
\hline \multirow[t]{3}{*}{ Variável } & \multicolumn{8}{|c|}{ Função exercida } & \multirow{3}{*}{$\mathbf{p}$} \\
\hline & \multicolumn{2}{|c|}{$\begin{array}{l}\text { Coordenador } \\
\text { de curso }\end{array}$} & \multicolumn{2}{|c|}{$\begin{array}{l}\text { Professor } \\
\text { supervisor }\end{array}$} & \multicolumn{2}{|c|}{$\begin{array}{c}\text { Coordenador } \\
\text { de estágio }\end{array}$} & \multicolumn{2}{|c|}{ Total } & \\
\hline & $\mathbf{n}$ & $\%$ & n & $\%$ & $\mathbf{n}$ & $\%$ & $\mathbf{n}$ & $\%$ & \\
\hline \multicolumn{10}{|c|}{ Utiliza TICs nos estágios para comunicação com } \\
\hline Alunos & 4 & 44,4 & 9 & 100 & 9 & 100 & 22 & 81,5 & $0,005^{*}$ \\
\hline Preceptores & 3 & 33,3 & 6 & 66,7 & 6 & 66,7 & 15 & 55,6 & 0,420 \\
\hline Docentes & 4 & 44,4 & 9 & 100 & 8 & 88,9 & 21 & 77,8 & $0,024 *$ \\
\hline Coordenador do curso & - & - & 8 & 88,9 & 8 & 88,9 & 16 & 88,9 & 1,000 \\
\hline Professor supervisor & 4 & 44,4 & - & - & 8 & 88,9 & 12 & 66,7 & 0,131 \\
\hline Coordenador do estágio & 4 & 44,4 & 8 & 88,9 & - & - & 12 & 66,7 & 0,131 \\
\hline PROACAD $* *$ & 3 & 33,3 & 2 & 22,2 & 6 & 66,7 & 11 & 40,7 & 0,226 \\
\hline \multicolumn{10}{|c|}{ Utiliza TICs nos estágios para outras finalidades } \\
\hline Divulgar informações & 4 & 44,4 & 8 & 88,9 & 9 & 100 & 21 & 77,8 & 0,024 \\
\hline Estratégias pedagógicas & 3 & 33,3 & 5 & 55,6 & 3 & 33,3 & 11 & 40,7 & 0,693 \\
\hline Pesquisas & 3 & 33,3 & 4 & 44,4 & 3 & 33,3 & 10 & 37,0 & 1,000 \\
\hline Total & 9 & 100 & 9 & 100 & 9 & 100 & 27 & 100 & \\
\hline
\end{tabular}

* Diferença significativa (5\%), teste Exato de Fisher

** Pró-Reitoria acadêmica da UFPE

Na tabela 3 estão apresentados os resultados referentes ao domínio no uso de TICs. Não se observou diferenças significativas $(\mathrm{p} \leq 0,05)$ entre os grupos para nenhuma das categorias das três variáveis estudadas.

A maioria da amostra total afirmou ter domínio mediano no uso das TICs, tendo o grupo de professores apresentado os menores valores. Apenas uma parcela da amostra total afirmou ter domínio baixo ou regular. Entretanto, a maioria afirmou não ter formação relacionada ao uso de TICs, sendo que todos dos coordenadores de ECS disseram não possuir nenhuma formação. Dentre os 5 respondentes que informaram não possuir formação em TICs a maioria era coordenador de curso. Sobre o emprego de métodos $\mathrm{EaD}$ voltados às atividades dos ECS, a maioria dos respondentes afirmou não utilizá-los. Entre os que afirmaram usar esse método, os maiores percentuais foram observados no grupo dos coordenadores de estágio e os menores entre os professores.

\section{DISCUSSÃO}

Os resultados sobre a caracterização da amostra estudada revelam que a maioria dos docentes é constituída por mulheres, assim como o observado em outros estudos semelhantes ${ }^{18,19}$. Esse achado é consistente com explicações acerca do processo histórico de inserção feminina no mercado de trabalho relacionado ao campo educacional ${ }^{20}$. Contudo, outro estudo sobre o perfil sociodemográfico de docentes da área da saúde de uma universidade pública do sul do Brasil mostrou que mais da metade deles era do sexo masculino ${ }^{19}$.

Sobre o padrão de utilização das TICs pelos docentes entrevistados nas atividades de ECS, verificou-se que a maioria deles, independente da função exercida, faz o uso de TICs. O menor uso de TICs pelos coordenadores de curso em relação aos demais docentes pode ser justificada pelo fato de caber aos professores e coordenadores dos estágios o desempenho de suas funções em contato direto com os alunos e os profissionais dos serviços de saúde nos 
quais os ECS são realizados. Esse resultado é coerente com as determinações oficiais formuladas para os ECS na saúde, que solicitam que o ensino e a supervisão tutorial que devem ser realizadas por docentes ${ }^{21}$.

Tabela 3. Domínio no uso de TICs, segundo a exercida

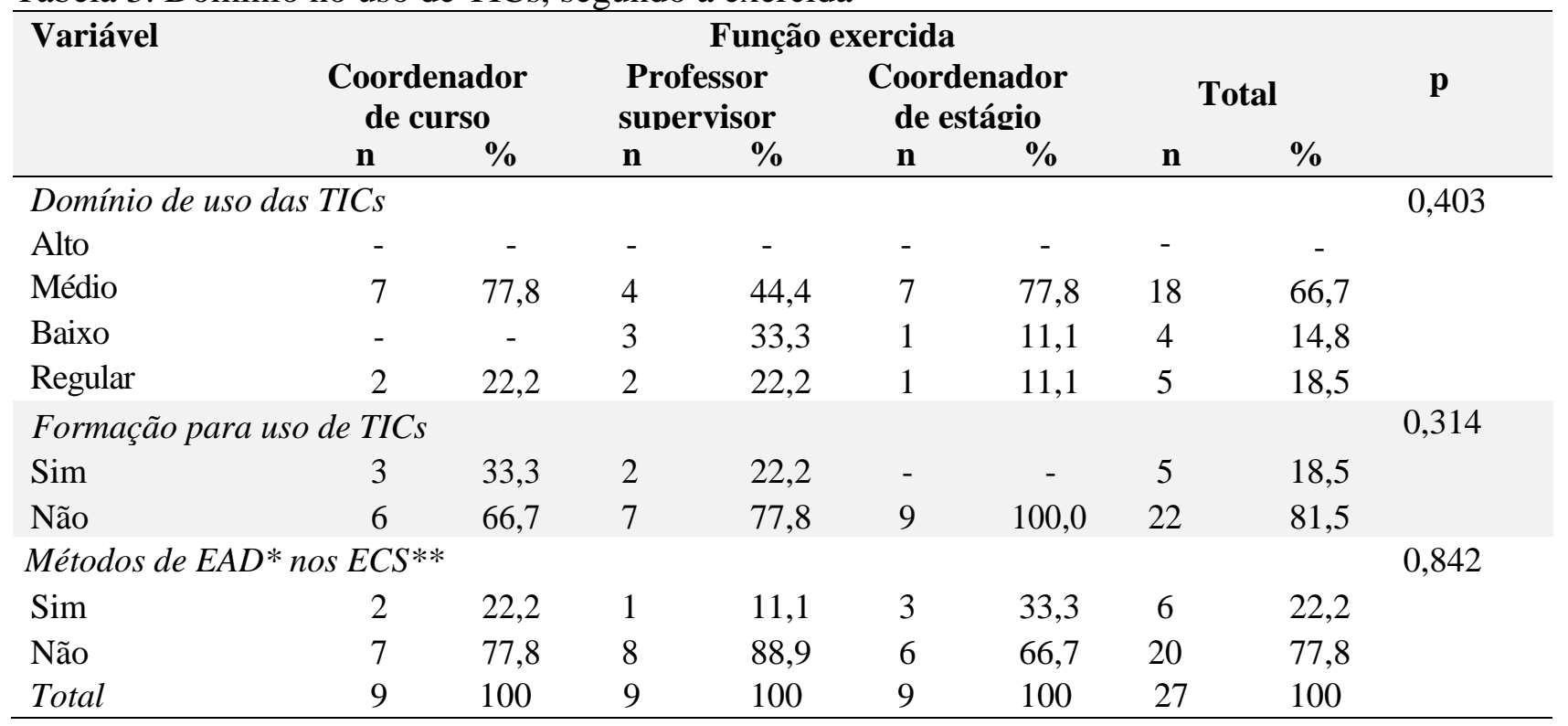

Teste Exato de Fisher

* Ensino a distância; **Estágios curriculares supervisionados

Da mesma forma, são os professores supervisores e coordenadores de estágio que utilizam com maior frequência os diferentes recursos de TICs, destacando-se em ordem de preferência o Facebook, o E-mail e o WhatsApp. Esperava-se, neste estudo, observar um maior uso do aplicativo WhatsApp ao invés do Facebook para a comunicação remota com os participantes dos ECS, uma vez que estudos informam que o WhatsApp é o recurso de comunicação mais utilizado entre os brasileiros na atualidade ${ }^{22}$. Contudo, outros autores ${ }^{23}$ discutem ser o WhatsApp uma rede mais reservada e de socialização mais íntima, pois para compartilhar dessa rede os usuários precisam partilhar entre si o número de seus telefones móveis. Já no Facebook, com apenas com um click o usuário adiciona amigos, conhecidos e conhecidos de conhecidos, com acesso às informações disponíveis nos perfis dos usuários.
Quanto à finalidade no emprego de TICs nas atividades dos docentes durante os ECS, observouse maior uso para a comunicação a distância, mais utilizada pelos professores e coordenadores de ECS na interação com alunos e preceptores. Tal resultado aponta uma incorporação do tipo de supervisão mediada por tecnologias digitais, além da presencial e concordâncias foram verificadas ${ }^{24}$. Outras aplicações das TICs com a intenção de qualificar a formação discente na rede SUS ${ }^{25}$ também foram verificadas, destacando-se a divulgação de informações e estratégias pedagógicas. Esses achados vão ao encontro de discussões sobre o potencial de uso das redes sociais com a finalidade pedagógica $^{25}$. Discute-se que o Facebook, assim como o Google+ e o LinkedIn possuem essas funcionalidades requeridas para serem utilizadas como ambientes virtuais de aprendizagem ${ }^{22,26}$.

A aplicação das mídias sociais como recurso 
educacional tem sido cada vez mais observada por usuários nos diferentes níveis de escolaridade. Mais especificamente na Odontologia, pesquisa recente revelou que, segundo os estudantes, a inserção do Facebook e do WhatsApp como ferramentas pedagógicas são positivas, uma vez que o Facebook pode ser empregado pela facilidade de acesso e obtenção de material didático publicado na página virtual da disciplina, enquanto o WhatsApp seria vantajoso na obtenção de informações sobre a disciplina de forma rápida ${ }^{27}$.

Diante disso, os resultados encontrados sobre maior preferência dos docentes quanto ao uso do facebook nas atividades dos ECS, sugerem que sejam promovidos estudos e avaliações institucionais sobre a viabilidade de implementação de AVA, com esse fim. Segundo Oliveira $(2007)^{28}$, as TICs são ferramentas importantes, capazes de ampliar as chances de aprendizagem do aluno e cuja incorporação no ensino é um fato irreversível. Contudo, Silva e Pereira $(2013)^{2}$ salientam que os ambientes virtuais devam ser adaptados não só ao perfil do corpo docente, mas também ao contexto de uso. Embora uma parcela dos entrevistados tenha informado utilizar métodos de $\mathrm{EaD}$, a maioria deles não os empregam. Mesmo com os avanços tecnológicos no campo da saúde e do ensino, os quais ampliaram as oportunidades do ensino à distância em ambientes virtuais distintos, onde se pode interagir e adquirir diferentes conhecimentos em tempo real ${ }^{29}$, a prática revela o pouco uso. As induções em políticas de educação permanente em saúde (EPS) no SUS consideram a possibilidade de utilização do EaD como um instrumento estratégico para formação dos profissionais da saúde, com a aplicação de recursos tecnológicos que possibilitem processos educativos mais interativos dentro e fora do serviço ${ }^{29}$.

Sublinha-se que foi criado, em 2005, o sistema Universidade Aberta do Brasil (UAB), integrado por universidades públicas e que oferece cursos de nível superior para populações com dificuldade de acesso à formação universitária, por meio do uso da metodologia da $\mathrm{EaD}^{30}$. A partir de 2007, a UFPE, deu início à implementação do primeiro curso de graduação a distância ${ }^{31}$. Contudo, esses movimentos institucionais parecem não ter ainda sensibilizado a maioria dos cursos da Área da Saúde quanto às vantagens do uso de modalidades $\mathrm{EaD}$ no âmbito da $\mathrm{UFPE}^{28}$. Dentre os que afirmaram usar $\mathrm{EaD}$, apenas um entrevistado informou utilizar o AVA institucional para desenvolver interações a distância com os alunos e preceptores dos serviços.

Destaca-se que a inserção de recentes inovações tecnológicas implicou em mudanças que exigem novas formas de construção do conhecimento que vão além da formação do professor $^{32,33}$. Com este interesse, investigou-se o domínio dos docentes quanto ao uso das TICs. A maioria afirmou ter um domínio mediano que foi obtido na prática. Esses achados são consistentes com outros autores que identificam deficiências no uso das TICs pelos profissionais do ensino, que são justificadas pelo fato de uma parcela expressiva de docentes em atuação profissional não ter nascido "digital", enquanto na atualidade os alunos tem avançada habilidade em TICs para acesso muito mais rápido e fácil às informações e para a interação entre pessoas a distância ${ }^{32,33}$. Nesta perspectiva, considera-se premente a aquisição dessas competências pelos docentes, o que lhes permitirá "reconfigurar", criativamente, uma determinada aplicação tecnológica para fins pedagógicos mais eficientes, inserindo os recursos tecnológicos emergentes em suas práticas de ensino, num ambiente interativo, focado agora no aluno ${ }^{15}$.

Sobre as limitações inerentes ao tipo de estudo adotado, foram tomados cuidados metodológicos com o intuito de que os resultados expressassem de fato a opinião prestada pelos participantes, mas devem ser considerados possíveis vieses de informação.

Espera-se que os achados revelados por este estudo contribuam para o aprimoramento dos 
processos formativos de docentes quanto ao uso mais competente das TICs no contexto educacional, estabelecendo uma rede dialógica de interação com o intuito de promover a ruptura do distanciamento entre sujeito e sociedade, promovendo continuamente a qualificação dos processos de integração ensino-serviço.

\section{CONCLUSÕES}

As TICs mostraram-se incorporadas com nuances variadas na integração ensino-serviço nos cursos da Área da Saúde da UFPE, com diferenças de aplicabilidade de acordo com a função exercida pelo docente nos estágios. Apesar da institucionalização de um AVA, observou-se uso de múltiplas ferramentas de uso geral. Apesar das TICs serem utilizadas pela maioria dos entrevistados, é necessário melhorar o domínio de uso desses recursos para potencializar as atividades dos ECS, com introdução dos métodos de $\mathrm{EaD}$ disponíveis na instituição.

\section{ABSTRACT \\ Incorporation of information and communication technologies in education- service integration of health courses from a public university}

With the objective of analyzing the incorporation of Information and Communication Technologies (ICT) by professors of the nine health courses of the Federal University of Pernambuco (UFPE) in teaching-service integration activities in Supervised Curricular Internships (SCI), an observational and descriptive study was conducted with a quantitative approach. The sample consisted of course and internships coordinators, as well as teachers supervising the SCI of those courses (27). For the data collection, an interview form was submitted to face validation and applied face to face. All 27 teachers selected were interviewed, being $92.6 \%$ female and $85.2 \%$ with a doctorate. The majority used ICT $(77.8 \%)$ in SCI, but differences were observed regarding choice $(\mathrm{p}=0.032)$. Facebook $(63.0 \%)$ was the most favored and most used ICT by teachers $(88.9 \%)$; followed by e-mail $(59.3 \%)$ and WhatsApp (37.0\%). The communication with students and preceptors was predominant on the ICT employment goals in the SCI, which was also more accomplished by the internship coordinators and teachers than by the coordinators of the courses $(\mathrm{p}=$ 0.024). The majority reported having a median domain in ICT and only $18.5 \%$ obtained training for their use. The majority $(77.8 \%)$ used distance education methods in SCI, despite the UFPE, to make institutional platforms available. The ICT have been incorporated with varied nuances in the teaching-service integration, with differences of applicability according to the function exerted by teacher in the internships.

Descriptors: Higher Education. Social Media. Information Technology, Internships. Unified Health System.

\section{REFERÊNCIAS}

1. Lobo ASM, Maia LCG. O uso das TICs como ferramenta de ensino aprendizagem no ensino superior. Cad Geografia. 2015;25 (44):16-26.

2. Neves CEB. Desafios da Educação Superior. Sociologias. 2007;(17):14-21.

3. Brasil. Ministério da Saúde. Ministério da Educação. Portaria interministerial $n^{\circ} 2.101$, de 3 de novembro de 2005. Institui o Programa Nacional de Reorientação da Formação Profissional em Saúde - PróSaúde - para os cursos de graduação em Medicina, Enfermagem e Odontologia. [Acesso em 14 dez. 2017]. Disponível em: https://www.portaleducacao.com.br/conteu do/artigos/odontologia/portaria-interminis terial-ms-mec-n-2101-de-3-de-novembrode-2005/3454

4. Brasil. Ministério da Saúde. Ministério da Educação. Portaria interministerial $n^{\circ} 917$, de 6 de maio de 2009. Estabelece orientações e diretrizes técnicoadministrativas para a execução do Programa de Educação pelo Trabalho para a 
Saúde - PET-Saúde. [Acesso em 14 dez. 2017]. Disponível em: http://bvsms.saude. gov.br/bvs/saudelegis/gm/2009/pri0917_06 _05_2009.html

5. Ceccim RB, Feuerwerker LO. Quadrilátero da formação para a área da saúde: ensino, gestão, atenção e controle social. Physis (Rio J). 2004; 14(1):41-65.

6. Assega ML, Lopes Jr LC, Santos EV. A interdisciplinaridade vivenciada no PETSaúde. Ciênc Saúde (Porto Alegre). 2010;3(1):29-33.

7. Brasil. Ministério da Saúde. Ministério da Educação. A aderência dos cursos de graduação em enfermagem, medicina e odontologia às diretrizes curriculares nacionais - 162 p.: il. - (Série F. Comunicação e Educação em Saúde). Brasília: Ministério da Saúde, 2006.

8. Brasil. Resolução $n^{\circ}$ 03/2002 de 19 de fevereiro de 2002. Dispõe sobre as Diretrizes Curriculares Nacionais dos cursos de graduação em odontologia. Diário Oficial da União 2002. [Acesso em 14 dez. 2017]. Disponível em: http://portal.mec.gov.br/cne/ arquivos/pdf/CES032002.pdf.

9. Badan DEC, Marcelo VC, Rocha DG. Percepção e utilização dos conteúdos de saúde coletiva por cirurgiões-dentistas egressos da Universidade Federal de Goiás. Ciênc Saúde Coletiva. 2010;15:1811-18.

10. Associação Brasileira de Ensino Odontológico (ABENO). Análise sobre as diretrizes curriculares nacionais para os cursos de graduação em odontologia. Rev ABENO. 2002; 2(1):35-8.

11. Lima TC, Paixão FRC, Cândido EC, Campos CJG, Ceolim MF. Estágio curricular supervisionado: análise da experiência discente. Rev Bras Enferm. 2014;67(1):133-140.

12. Benito GAV, Tristao KM, Paula ACSF,
Santos MA, Ataide LJ, Lima RCD. Desenvolvimento de competências gerais durante o estágio supervisionado. Rev Bras Enferm. 2012;65(1):172-8.

13. Mendes A. TIC - Muita gente está comentando, mas você sabe o que é? [Acesso em 27 fev. 2017]. Disponível em: https://imasters.com.br/artigo/8278/gerencia-deti/tic-muita-gente-esta-comentando-mas-vocesabe-oque $/$ ?trace $=1519021197 \&$ source $=$ single. .

14. Feldkercher N, Mathias CV. Uso das TICs na Educação Superior presencial e a distância: a visão dos professores. Rev Iberoam Tecnol Educ Educ Tecnol. 2011;6(6):84-92.

15. Silva MAD, Dias da Silva MA, Pereira AC. Utilização das TIC no ensino complementar da histologia nas faculdades de odontologia do estado de São Paulo. Scientia Plena. 2013; 9:1-7.

16. UNESCO. World Education Report, 1998, p. 19-20. [Acesso em 27 fev. 2017]. Disponível em: http://unesdoc.unesco.org/images/0011/001108/ $110875 \mathrm{eb} . p d f$.

17. Boynton PM, Greenhalgh T. Selecting, designing, and developing your questionnaire. BMJ. 2004;328(7451):1312-5.

18. Dávila MHX, Casagrande RJT, Pereira VCG. Qualidade de vida do trabalhador de uma instituição de ensino. Cad Esc Saúde. 2010;4(1):110-26.

19. Santos MN, Marques AC. Condições de saúde, estilo de vida e características de trabalho de professores de uma cidade do sul do Brasil. Ciênc Saúde Colet. 2013;18(3):837-46.

20. Souza SM. Caracterização sociodemográfica de docentes da área da saúde. Renome. 2015,4(1):15-28.

21. Ferreira RC, Fiorini VML, Crivelaro E. Formação profissional no SUS: o papel da Atenção Básica em Saúde na perspectiva 
docente. Rev Bras Educ Méd. 2010;34(2):207-15.

22. Rodrigues TA. Utilização do aplicativo whatsapp por professores em suas práticas pedagógicas. 2015. Simpósio Hipertexto e Tecnologias na Educação, 6. In: Anais, Recife, 2015.

23. Ferreira NS, Arruda Filho EJM. Facebook e whatsapp: uma análise das preferências de uso. Reuna. 2015;20(3):47-64.

24. Piconez SCB, Filatro AC. O desenvolvimento profissional da docência na formação de professores face a utilização das tecnologias. Educação Temática Digital. 2009;10(2):394.

25. Cavalheiro MTP, Aloide LG. Formação para o SUS e os desafios da integração ensino serviço. Cad FNEPAS. 2011;1:1-9.

26. Possolli GE, Nascimento GL, Silva JOM. A Utilização do facebook no contexto acadêmico: o perfil de utilização e as contribuições pedagógicas e para educação em saúde. Renote. 2015;13(1):322-33.

27. Souza FB, Lopes MGQ, Lima Filho RM. Redes sociais na aprendizagem em odontologia: opinião dos estudantes de uma universidade brasileira. Rev Cuba Estomatol. 2017;54(2):1-12.

28. Oliveira MAN. Educação à distância como estratégia para a educação permanente em saúde: possibilidades e desafios. Rev Bras Enferm. 2007;60(5):585-9.

29. Silva AN. Limites e possibilidades do ensino à distância $(\mathrm{EaD})$ na educação permanente em saúde: revisão integrativa. Ciênc Saúde Colet. 2015;20(4):1099-07.

30. Osório MRV, Garcia MMA. Universidade Aberta do Brasil (UAB): (re) modelando o território da formação de professores. Cad Educ. 2011;(38):119-49.

31. Tavares LD. Desafios na criação da cultura de EaD na UFPE. Eutomia. 2015;1(7):241-53.

32. Joly MCRA, Silva BD, Almeida LS. Avaliação das competências docentes para utilização das tecnologias digitais da comunicação e informação. Currículo sem Fronteiras. 2012;12(3):83-96.

33. Ponte JP. Tecnologias de informação e comunicação na formação de professores: que desafios? Rev Iberoam Educ. 2000;(24):63-90.

Correspondência para:

Emille Raíza Luna Gomes Ramos

e-mail: mly_ramos@ hotmail.com

Rua João de Lima, 46/202 Iputinga

506702-90 Recife/PB 